\title{
Questes
}

\section{Trouver la paix : introduction}

\section{Stéphanie Richard et Irène Strobbe}

\section{(2) OpenEdition}

\section{Journals}

Édition électronique

URL : http://journals.openedition.org/questes/153

DOI : 10.4000/questes.153

ISSN : 2109-9472

\section{Éditeur}

Les Amis de Questes

\section{Édition imprimée}

Date de publication : 25 septembre 2013

Pagination : 9-26

ISSN : 2102-7188

\section{Référence électronique}

Stéphanie Richard et Irène Strobbe, «Trouver la paix : introduction », Questes [En ligne], 26 | 2013, mis en ligne le 01 janvier 2014, consulté le 23 septembre 2020. URL : http://journals.openedition.org/ questes/153 ; DOI : https://doi.org/10.4000/questes.153 


\title{
Introduction
}

\section{Stéphanie RICHARD et Irène STROBBE}

\author{
Université Paris-Sorbonne (Paris IV)
}

\begin{abstract}
Ainsi donc, la paix du corps, c'est l'agencement harmonieux de ses parties; la paix de l'âme sans raison, c'est le repos bien réglé de ses appétits ; la paix de l'âme raisonnable, c'est l'accord bien ordonné de la pensée et de l'action; la paix de l'âme et du corps, c'est la vie et la santé bien ordonnées de l'être animé ; la paix de l'homme mortel avec Dieu, c'est l'obéissance bien ordonnée dans la foi sous la loi éternelle ; la paix des hommes, c'est leur concorde bien ordonnée ; la paix de la maison, c'est la concorde bien ordonnée de ses habitants dans le commandement et l'obéissance; la paix de la cité, c'est la concorde bien ordonnée des citoyens dans le commandement et l'obéissance ; la paix de la cité céleste, c'est la communauté parfaitement ordonnée et parfaitement harmonieuse dans la jouissance de Dieu et dans la jouissance mutuelle en Dieu; la paix de toutes choses, c'est la tranquillité de l'ordre. L'ordre, c'est la disposition des êtres égaux et inégaux, désignant à chacun la place qui lui convient ${ }^{1}$.
\end{abstract}

Voilà comment l'évêque d'Hippone définissait la paix dans La Cité de Dieu. Force de cohésion universelle, la paix correspond alors à la

\footnotetext{
${ }^{1}$ Euvres de saint Augustin. 33-37, La Cité de Dieu, 37 Livres XIX-XXII, texte de la $4{ }^{\mathrm{e}}$ édition de Bernard Dombart et Alphonse Kalb, introduction et notes par Gustave Bardy, traduction française de Gustave Combès, Paris, Desclée de Brouwer, 1960, XIX, 13, p. 109 et 111. Voir Marie-Josèphe Rondeau, «Le tournant chrétien. La paix, quelle paix ? L'enseignement de la Cité de Dieu ", dans Les fondements de la paix, Des origines au début du XVIII siècle, Actes du colloque du Mémorial de Caen et du CRHQ (CNRS et Université) de Caen, dir. Pierre Chaunu, Paris, PUF, 1993, p. 83-94, p. 88-89.
} 
tranquillitas ordinis, la tranquillité de l'ordre, à la stabilité induite par le respect de la hiérarchie où l'inférieur reste toujours subordonné au supérieur.

Cette définition, loin de constituer l'aboutissement de toute réflexion sur la paix, fut pour les hommes du Moyen Âge le point de départ - ou tout au moins, la pierre angulaire - de la plupart de leurs méditations autour du sujet. Aussi, à la suite de saint Augustin, de nombreux théologiens, clercs, chroniqueurs mais aussi des gouvernants reprirent-ils les réflexions de l'évêque en les modulant. À la multiplicité des types de paix ou de leurs fondements répond la pluralité des définitions. $\mathrm{Au} \mathrm{XI}^{\mathrm{e}}$ siècle, pour Guigues I ${ }^{\mathrm{er}}$ le Chartreux, la paix résulte de la reconnaissance de la vérité2 Bernard de Clairvaux, quant à lui, distingue quatre sortes de paix liées aux quatre vertus cardinales ${ }^{3}$, tandis que Pierre le Chantre propose une division tripartite et graduée de la paix : paix temporelle (fausse et trompeuse), paix du cœur (vraie mais préparatoire), paix de l'éternité ${ }^{4}$.

On le voit, le terme « paix » ne répond pas à une définition unique et simple. Les hommes du Moyen Âge en avaient eux-mêmes bien conscience, jouant à l'occasion sur cette multiplicité de sens, une multiplicité et une complexité sur lesquelles les Questeurs ont choisi de mener une réflexion au cours de trois séminaires intitulés «Trouver la paix ». Dans cette perspective, nous distinguerons de prime abord deux genres de paix : la paix entre les hommes, et celle que nous appelerons « la paix intérieure » et dont il conviendra de préciser le sens ultérieurement.

2 Voir Guigues I ${ }^{\mathrm{er}}$ prieur de Chartreuse, Les Méditations (recueil de pensée), Introduction, texte critique, traduction et notes par un chartreux, Paris, Éditions du Cerf, Sources Chrétiennes $\mathrm{n}^{0} 308$ (série des textes monastiques d'Occident $\mathrm{n}^{\circ} \mathrm{LI}$ ), 1983, méditations 128, 144 et 165, p. 143, 147 et 165.

3 Voir Sententiarium Series Prima 29 dans Bernard de Clairvaux, Sermones, III Sententiae, éd. J. Leclercq et H. Rochais, Romae, Editiones Cistercenses, 1972, p. 17.

${ }^{4}$ Voir capitula 100 «De pace» dans Petri Cantoris Parisiensis, Verbum Adbreviatum, Textus Prior, éd. Monique Boutry, Turnhout, Brepols, 2012, p. 558-563 (p. 558-559).

${ }^{5}$ Nicolas Offenstadt, Faire la paix au Moyen Âge, Paris, Odile Jacob, 2007, p. 13-14. 
Entamons notre recherche avec ce qui pourrait apparaître le plus évident et qui nécessite sans doute le plus de prudence : la paix entre les individus.

\section{De l'absence de guerre à la concorde entre les individus}

Fin des troubles ou lien actif entre les individus? La paix ne se laisse pas appréhender facilement et le caractère protéiforme de cette notion serait au moins en partie responsable du manque d'intérêt ancien dont elle fit l'objet parmi les médiévistes ${ }^{6}$; la guerre au contraire, comme tissu d'événements plus immédiatement préhensible et délimitable, a suscité la production d'une abondante littérature critique portant sur les batailles, l'armée, les fortifications ou encore les codes de chevalerie. Il faut cependant nuancer ce propos: si l'étude de la paix a été longtemps délaissée en France, l'historiographie allemande et anglophone, appuyée sur des ancrages institutionnels comme la Friedensforschung et les Peace Studies, s'est emparée du sujet dès les années 1950.

En France, jusque dans les années 1980, la paix n'apparaît que de façon secondaire dans les recherches, le plus souvent sous un angle diplomatique ou à travers l'étude de l'arbitrage des papes ${ }^{7}$. Exception notable toutefois, en 1939, Roger Bonnaud-Delamare choisit de s'interroger sur l'idée de paix à l'époque carolingienne ${ }^{8}$. Il réfléchit sur les liens unissant guerre et paix en montrant qu'au Moyen Âge, la paix ne

\footnotetext{
6 Voir par exemple Nicolas Offenstadt, op. cit., p. 25 et Diane Wolfthal, "Introduction », dans Peace and Negotiation: Strategies for Coexistence in the Middle Ages and the Renaissance, éd. Diane Wolfthal, Turnhout, Brepols, coll. «Arizona Studies in the Middle Ages and the Renaissance », 4, 2000, p. xi-xxviii (p. xv).

${ }^{7}$ Voir par exemple Charles Petit-Dutaillis et Paul Collier, « La diplomatie française et le traité de Brétigny », Le Moyen Âge, I, janvier-février. 1897, p. 1-35 ; Guillaume Mollat, " Innocent VI et les tentatives de paix entre la France et l'Angleterre », Revue d'histoire ecclésiastique, X, 1909, p. 729-743 ; Jean Gaudemet, "Le rôle de la papauté dans le règlement des conflits entre États aux XIII ${ }^{\mathrm{e}}$ et $\mathrm{XIV}^{\mathrm{e}}$ siècles ", dans Recueils de la société Jean Bodin, La Paix, Bruxelles, Éditions de la Librairie Encyclopédique, t. XV, 1961, p. 79-106 ; etc. Les études de ce genre sont nombreuses.

${ }^{8}$ Roger Bonnaud-Delamare, L'idée de paix à l'époque carolingienne, Paris, Éditions Domat-Monchrestien, 1939.
} 
représente pas la négation de la guerre : au contraire, c'est plutôt la guerre qui est la négation de la paix. Les affrontements armés ne sont d'ailleurs qu'une forme de négation de la paix parmi d'autres, celle-ci pouvant également être troublée par les famines, la maladie ou encore les hérésies. Par ailleurs, on trouve chez plusieurs auteurs médiévaux l'idée selon laquelle la guerre est un moyen d'imposer ou de restaurer la paix : au $\mathrm{VII}^{\mathrm{e}}$ siècle, Isidore de Séville fait de la paix l'un des quatre stades de la guerre 9 . Six siècles plus tard, le même type de conception prévaut chez Guillaume Durand puisque, suivant l'évêque de Mende, le célébrant de l'adoubement chevaleresque doit conseiller à l'adoubé d'être un miles pacificus, un "guerrier pacifique " recherchant au sein même des combats la conclusion d'une paix juste et évitant toute violence inutile ${ }^{10}$. L'historiographie récente souligne bien que guerre et paix, loin d'être des concepts strictement antithétiques, peuvent apparaître complémentaires. Songeons, dans cette perspective, aux justifications de la croisade avancées par les prédicateurs, pour qui la paix de la Chrétienté doit permettre de mener la guerre contre les Infidèles; en 2002, Tomaž Mastnak a analysé la façon dont les mouvements de paix du XI ${ }^{\mathrm{e}}$ siècle, en cherchant à pacifier la Chrétienté occidentale, ont en même temps influencé les relations du monde chrétien vers l'extérieur en faisant des musulmans l'ennemi ${ }^{11}$. Dans un autre domaine, Jenny Benham s'est interrogée sur les accords par lesquels les gouvernants décident de se promettre une aide mutuelle contre

\footnotetext{
${ }^{9}$ Avec le combat (pugna), la fuite (fuga) et la victoire (victoria).Voir San Isidoro de Sevilla, Etimologías, Edición bilingüe por Jose Oroz Reta y Manuel A. Marcos Casquero, Madrid, La editorial católica, coll. «Biblioteca de autores cristianos », 18821883, t. II, (Etymologiarum Liber XVIII, 1. De bellis, 11), p. 384.

10 Voir Franco Cardini, "Guerre et croisade», dans Dictionnaire raisonné de l'Occident médiéval, dir. Jacques Le Goff, Jean-Claude Schmitt, Paris, Fayard, 1999, p. 435-449, p. 438.

11 Tomaž Mastnak, Crusading Peace. Christendom, The Muslim World, and Western Political Order, Berkeley/Los Angeles/London, University of California Press, 2002.
} 
leurs ennemis respectifs ${ }^{12}$ : si ces accords pourraient être de nos jours qualifiés d'alliance, le langage qui y est utilisé, plaçant la paix au cœur des préoccupations, permet selon l'auteur de les catégoriser comme traités de paix. La cessation des hostilités dans un espace donné favorise alors le déclenchement, la reprise ou la perpétuation des affrontements dans un autre. L'intrication très forte entre guerre et paix pousse aujourd'hui de nombreux chercheurs à ne plus détacher l'étude de la paix de celle des conflits: les ouvrages et les colloques internationaux les plus récents affichent leur volonté d'articuler plutôt que de séparer ces deux objets ${ }^{13}$.

Si la paix ne peut se réduire à être le contraire de la guerre, c'est peut-être parce qu'en réalité elle correspond à une notion plus positive que la seule absence de trouble. Envisagée par saint Augustin comme la restauration de l'harmonie de la création de Dieu, sa réalisation demande en effet bien plus que le simple arrêt des combats. Pour rétablir un ordre juste, il est nécessaire d'obtenir réparation de l'injustice subie et éventuellement un dédommagement des conséquences induites par les conflits. Dans ce cadre, la paix peut revêtir un caractère clairement volontaire : certaines définitions accordées au mot pax vont en ce sens, le terme pouvant en effet désigner un groupe de personnes ou une association dont le but est le maintien de l'ordre ${ }^{14}$. Dans une perspective proche, la paix médiévale représente plus largement selon Tom B. Lambert un moyen de définir un groupe à l'intérieur duquel les membres ont une obligation

\footnotetext{
12 Jenny Benham, Peacemaking in the Middle Ages : principles and practice, Manchester/New York, Manchester University Press, 2011, p. 8-9.

13 Voir par exemple l'ouvrage Frieden schaffen und sich verteidigen im Spätmittelalter/Faire la paix et se défendre à la fin du Moyen Âge, dir. Gisela Naegle, München, Oldenbourg Verlag, coll. «Pariser historische Studien », 2012, et le colloque «Guerre et paix dans le Proche-Orient médiéval, $\mathrm{X}^{\mathrm{e}}-\mathrm{XV}^{\mathrm{e}}$ siècle » qui s'est tenu le 15 décembre 2011 au Caire.

${ }^{14}$ Voir l'entrée « Pax » dans Jan Frederik Niermeyer, Mediae Latinitatis Lexicon Minus, Leiden, E. J. Brill, 1976 : sens 15 : « association qui se donne pour but d'effectuer une régulation de la paix », et sens 20 : «association pour le maintien de la paix urbaine, la commune », p. 778-779.
} 
positive d'aider l'autre, sur le modèle de la parenté ${ }^{15}$. Qu'elle soit restauration de l'ordre ou action collective volontaire, la paix selon ces conceptions est donc beaucoup plus active que la seule absence de guerre.

A partir des années 1980, l'analyse de la paix a commencé à se renouveler par le biais des études du règlement des conflits. Dans ce champ, à nouveau, la paix n'est pas le cœur du sujet : si la question est généralement abordée, c'est à travers l'examen des motivations animant les acteurs en présence, des résultats des différents processus de négociation ou encore des considérations sur la justice, que les théoriciens du Moyen Âge lient étroitement à la paix ${ }^{16}$. Cependant, l'adoption de cet angle $\mathrm{d}$ 'approche a permis de réaliser des avancées significatives. Les recherches portant sur la résolution des conflits, influencées à la fois par l'anthropologie juridique et par les travaux des médiévistes étrangers inspirés de cette même anthropologie juridique ${ }^{17}$, ont en effet conduit les historiens à considérer non plus seulement les formes institutionnelles de règlement des litiges, mais également les formes de l'infrajudiciaire ${ }^{18}$. Ils ont ainsi mis en avant le rôle des compromis permettant de régler les conflits, et par suite, tout en ménageant les intérêts matériels ou symboliques des parties, de revenir à

${ }^{15}$ Tom B. Lambert, «Introduction : Some Approaches to Peace and Protection in the Middle Ages ", dans Peace and Protection in the Middle Ages, dir. Tom B. Lambert, David Rollason, Durham, Toronto, Centre of Medieval and Renaissance Studies, Pontifical Institute of Medieval Studies, 2009, p. 1-16, p. 6.

${ }^{16}$ Le thème de l'union entre justice et paix comme fondement nécessaire du bon gouvernement a été développé dès saint Augustin. Relayé par les Etymologies d'Isidore de Séville, il a ensuite été développé tout au long du Moyen Âge à partir de différents supports (écrits de juristes, comme Jean Bouteiller, préambules des ordonnances des rois de France...). Voir sur ce sujet Claude Gauvard, "Justice et paix », dans Dictionnaire raisonné de l'Occident médiéval, op. cit., p. 587-594.

${ }^{17}$ Voir par exemple Patrick J. Geary, «Vivre en conflit dans une France sans État : typologie des mécanismes de règlement des conflits (1050-1200)», dans Annales. Économies, Sociétés, Civilisations. $41^{e}$ année, $\mathrm{n}^{\circ}$ 5, 1986, p. 1107-133; The settlement of disputes in early Medieval Europe, dir. Wendy Davies, Paul Fouracre, Cambridge/London, Cambridge University Press, 1986; ainsi que les travaux de Stephen D. White et de Gerd Althoff.

${ }^{18}$ Soit la part des initiatives privées pour mettre fin à une querelle. 
une situation pacifiée ${ }^{19}$. Certains résultats, tant français qu'étrangers, sont aujourd'hui partiellement remis en cause : Bruno Lemesle, par exemple, critique l'opinion selon laquelle la recherche de la paix est intrinsèque à toute querelle, car cette perspective, selon lui, conduit à oublier la part des litiges non résolus ${ }^{20}$. Toutefois, les études portant sur le règlement des conflits ont permis de réévaluer l'idée selon laquelle le Moyen Âge, et plus particulièrement la période féodale, a été un temps de violence anarchique $^{21}$. Elles ont eu des répercussions sur la façon dont les historiens ont pu considérer la paix et la façon de faire la paix à l'époque médiévale.

Dans les années 1990, en France, la paix est ainsi devenue un objet d'étude à part entière. Certes, différentes formes de paix avaient déjà été étudiées : la paix de Dieu et son corollaire la trêve de Dieu avaient fait l'objet de recherches approfondies dès 1857 par Ernest Sémichon, qui voyait dans les mouvements de paix l'origine des communes urbaines et diocésaines $^{22}$. La réflexion à ce sujet a été profondément renouvelée à la fin des années 1990 par Dominique Barthélémy, qui a combattu l'idée selon laquelle la paix et la trêve de Dieu témoignent d'une crise sociale au tournant du siècle ${ }^{23}$. Peu à peu, pour l'espace français en tout cas, s'esquisse une forme de chronologie de l'idée de paix en fonction de ceux

${ }^{19}$ Frederik Cheyette, «Suum cuique tribuere », French Historical Studies, 6, 1970, p. 287-299.

${ }^{20}$ Bruno Lemesle, Conflits et justice au Moyen Âge, Paris, Le Nœud Gordien, 2008, p. 288.

${ }^{21}$ Voir par exemple Stephen Weinberger, «Les conflits entre clercs et laïcs dans la Provence du XI ${ }^{\mathrm{e}}$ siècle », Annales du Midi, vol. 92, 1980, p. 369-379; Lester Little, "Formules monastiques de malédiction aux $\mathrm{IX}^{\mathrm{e}}$ et $\mathrm{X}^{\mathrm{e}}$ siècles ", Revue Mabillon, vol. 58, 1970-1975, p. 377-399 ; Henri Platelle, « Crime et châtiment à Marchiennes : étude sur la conception et le fonctionnement de la justice d'après les Miracles de sainte Rictrude (XII ${ }^{\mathrm{e}}$ siècle) », Sacris erudiri, vol. 24, 1980, p. 155-202.

${ }^{22}$ Voir Ernest Sémichon, La paix et la trêve de Dieu, Paris, Didier, 1857.

${ }^{23}$ Dominique Barthélémy, L'an mil et la paix de Dieu : la France chrétienne et féodale 980-1060, Paris, Librairie Arthème Fayard, 1999. 
qui doivent en être les promoteurs ${ }^{24}$. Après la période carolingienne au cours de laquelle le roi est perçu comme le garant de la paix publique, ce sont les assemblées et conciles de paix qui, à partir du $\mathrm{X}^{\mathrm{e}}$ siècle, par l'intermédiaire de la paix et de la trêve de Dieu, sont chargées d'éliminer la violence non contrôlée par le pouvoir royal ou les seigneurs. Au XII ${ }^{\mathrm{e}}$ siècle cependant, l'idéologie du roi protecteur de la paix du royaume retrouve sa force, et elle est développée au cours des siècles suivants à travers les miroirs des princes; en parallèle, le rôle du pape comme arbitre dans les conflits internationaux internes à la Chrétienté est également valorisé. Il ne faudrait pourtant pas en conclure que ce champ est clos : la théorisation de l'idée de paix est toujours étudiée aujourd'hui à travers les écrits des juristes, des chroniqueurs ou des théologiens ${ }^{25}$. La recherche française explore également de nouvelles directions, en examinant la production des discours de paix et les rituels ${ }^{26}$.

Les recherches allemandes et anglo-saxonnes n'ont d'ailleurs pas été en reste. Les théories médiévales sur la paix, les mouvements de paix et de trêve de Dieu, ou encore la question du règlement des conflits ont également donné lieu à des enquêtes approfondies. Aujourd'hui, les analyses se diversifient : outre des études variées, portant par exemple sur les liens entre paix et protection des individus ${ }^{27}$, les mêmes thématiques qu'en France sont explorées, parfois depuis plus longtemps. Ainsi, en Allemagne, les travaux de Gerd Althoff ont permis de s'interroger sur les

\footnotetext{
${ }^{24}$ Henri Dubois, "La paix au Moyen Âge», dans Les fondements de la paix, Des origines au début du XVIII siècle, Actes du colloque du Mémorial de Caen et du CRHQ (CNRS et Université) de Caen, dir. Pierre Chaunu, Paris, PUF, 1993, p. 95-108.

${ }^{25}$ Par exemple, dans le colloque dirigé par Gisela Naegle, les communications proposées dans le cadre de la troisième partie «Penser la paix ou faire la guerre?» portent sur cette question. Voir Frieden schaffen..., op. cit.

${ }^{26}$ Voir notamment les travaux de Nicolas Offenstadt, dont l'objectif est d'analyser les discours de paix comme une «mise en scène de soi», et de reconstituer une " grammaire rituelle de la paix » à partir des rituels (Nicolas Offenstadt, op. cit., p. 17 et p. 24).

${ }^{27}$ Peace and Protection..., op. cit.
} 
rituels de paix ${ }^{28}$. Dans le monde anglophone, Kiril Petkov s'est concentré sur un rituel spécifique, le baiser de paix, outil stratégique de la paix médiévale ${ }^{29}$. Ben Lowe a proposé une analyse conjointe de l'idée et des discours de paix dans l'Angleterre de la fin du Moyen Âge et du début de l'époque moderne : selon lui, ces discours ont émergé et évolué au cours même de débats portant sur des conflits spécifiques ; en retour, l'extension du répertoire des idées pacifistes a influencé l'idéologie de la guerre juste ${ }^{30}$. Par ailleurs, en France comme à l'étranger, la question des acteurs de la paix n'a pas été abandonnée: en dehors de la fonction pacificatrice habituellement attribuée aux gouvernants - ou aux femmes de leur entourage $^{31}$ - on s'interroge désormais sur le rôle des villes et des ligues de ville, en particulier aux $\mathrm{XIV}^{\mathrm{e}}$ et $\mathrm{XV}^{\mathrm{e}}$ siècles, ainsi que sur le rôle des intermédiaires (messagers, ambassadeurs) dans les processus de paix ${ }^{32}$.

A côté de ces avancées, l'étude de la paix semble pourtant accuser encore certains manques. Rares en effet sont les analyses portant sur les images de la paix ${ }^{33}$. Par ailleurs, l'idée de paix dans la littérature a reçu une attention moindre que l'histoire intellectuelle de la paix, en dehors des recherches d'Albrecht Hagenlocher sur la littérature germanique ${ }^{34}$, de

28 Voir par exemple Gerd Althoff, Spielregeln der Politik im Mittelalter. Kommunikation in Frieden und Fehde, Darmstadt, Primus Verl., 1997.

${ }^{29}$ Kiril Petkov, The Kiss of Peace, Ritual, Self and Society in the High and Late Medieval West, Leyde/Boston, Brill, 2003.

${ }^{30}$ Ben Lowe, Imagining Peace, A History of Early English Pacifist Ideas, 1340-1560, University Park, Pennsylvania State University Press, 1997.

${ }^{31}$ Nicolas Offenstadt, «Les femmes et la paix à la fin du Moyen Âge : genre, discours, rites » dans Le règlement des conflits au Moyen Âge, XXXI ${ }^{e}$ Congrès de la SHMESP (Angers, juin 2000), Paris, Publications de la Sorbonne, 2001, p. 317-333.

${ }^{32}$ Voir Pierre Monnet, « Conclusion », dans Frieden schaffen ..., op. cit., p. 357-372.

${ }^{33}$ On peut toutefois signaler l'article suivant: Klaus Arnold, «Friedensallegorien und bildliche Friedensappelle im späteren Mittelalter und in der Frühen Neuzeit », dans Krieg und Frieden im Übergang vom Mittelalter zur Neuzeit: Theorie, Praxis, Bilder (Guerre et paix du Moyen Âge au temps modernes: théories, pratiques, représentations), dir. Heinz Duchhardt et Patrice Veit, Mainz, Verlag Philipp von Zabern, 2000, p. 13-34.

${ }^{34}$ Albrecht Hagenlocher, Der « guote vride » : idealer Friede in deutscher Literatur bis ins frühe 14. Jahrhundert, Berlin, W. De Gruyter, 1992. 
Robert Yaeger sur le pacifisme de Geoffrey Chaucer et de John Gower ${ }^{35}$, ou encore de Tania van Hemelryck sur la femme et la paix dans la littérature ${ }^{36}$.

\section{Paix entre les individus et paix intérieure : continuité et enchâssement}

Rappelons ici les paroles de saint Augustin : « la paix de l'âme sans raison, c'est le repos bien réglé de ses appétits; la paix de l'âme raisonnable, c'est l'accord bien ordonné de la pensée et de l'action ${ }^{37}$. En nous appuyant sur cette définition partielle, c'est ce que nous appellerons ici la "paix intérieure ». Si l'évêque d'Hippone propose une définition de la paix où toutes les paix, depuis celle du corps et de l'âme à celle qui doit régner entre les citoyens, sont enchâssées, la recherche s'est peu penchée sur la paix intérieure en elle-même, excepté dans le cadre de la résolution de conflits interpersonnels : de la paix de Dieu à la paix des ménages ${ }^{38}$, les prédispositions internes des protagonistes sont mises en avant et les différences qui surgissent pourraient être davantage une question d'échelle que de nature. Ainsi, Dominique Barthélemy rapproche la pacification d'une faide par des moines de l'abbaye de Lobbes en 1060 d'une séance d'exorcisme : le diable quitte le cercle sacré tracé par les moines sous la forme d'un chien noir suivi des quelques belligérants qui, possédés par le démon, ne peuvent se résoudre à l'arrêt des combats fratricides ${ }^{39}$.

\footnotetext{
${ }^{35}$ Robert F. Yaeger, «Pax Poetica: On the Pacifism of Chaucer and Gower », Studies in the Ages of Chaucer, 9, 1987, p. 87-21.

${ }^{36}$ Tania Van Hemelryck, "La femme et la paix. Un motif pacifique de la littérature française médiévale ", dans Revue belge de philologie et d'histoire, vol. 84, $\mathrm{n}^{\circ}$ 2, 2006, p. 243-270.

${ }^{37}$ Voir supra note 1.

38 Julie Claustre, "Assurer la paix des "ménages". De la dette à la paix (Paris, XIV"XV ${ }^{\mathrm{e}}$ siècles) », dans Frieden schaffen..., op. cit., p. 199-214.

${ }^{39}$ « Il y avait là des chevaliers dont les inimitiés étaient telles que nul mortel ne pouvait arranger la paix entre eux. Pendant les années précédentes, les séditions excitées par le diable avaient fait perdre à des pères leurs fils, à des fils leurs pères, à des frères leurs
} 
Cependant, la noirceur des âmes n'influe pas seulement dans les négociations menées sur les champs de bataille. Elle revêt aussi un aspect capital dans la vie quotidienne où une affaire de cœur peut tourner à l'affrontement dans le sang pour des questions d'honneur. Là encore, le caractère paisible des individus est primordial dans le déclenchement et l'apaisement des conflits. Claude Gauvard, dans sa thèse, insiste sur le fait qu'il « existe deux sortes de sujets parfaitement antithétiques: les uns irradient la paix, les autres sèment la discorde ${ }^{40} »$. L'exemple qu'elle propose est celui d'un meunier qui a poursuivi de ses assiduités la future épouse d'un boulanger : il trouble ainsi l'ordre religieux mais aussi l'ordre social en étant l'instigateur du bruyant charivari qu'il réserve au couple finalement marié. Pour Claude Gauvard, « ce charivari n'est que la forme extériorisée du plaisir coupable de celui qui est "allé et venu entour elle en

frères. Quand le saint provoqua un afflux de gens vers lui, tous ceux-là vinrent aussi. Ceux que les dissensions épargnaient les exposèrent. Nous nous mîmes donc à discuter séparément les uns avec les autres. Il fallait que chacun donne à Dieu et au saint sa querelle, afin que le nombre de morts cesse de s'accroître. Certains y consentirent, à contrecœur, mais par crainte de Dieu et pour l'amour du saint. Mais une minorité persistait dans le refus. Nul ne parvenait à les fléchir. "Eh bien, dit le doyen Baudouin, portons le saint tout autour d'eux. Il faudra alors qu'ils consentent à la paix, suivant notre conseil, ou que leur dissidence les sépare de notre société, et qu'ils suivent le diable leur seigneur". Alors nous levâmes de terre le saint et nous commençâmes notre procession circulaire, avec prudence et en priant. Aussitôt il arriva ceci : les ennemis de la paix s'écartèrent, sans savoir ce que les nôtres avaient médité entre eux. L'idée reposait en effet sur la protection [fiducia] du saint que nous suivions. Et ceux qui pourraient une fois être inclus dans le cercle décrit par la procession, la puissance du diable ne prévaudrait plus. Sans tarder, la ruse du diable fut démasquée. Un chien noir passa entre eux et nous, et il montra ainsi qu'il les attirait loin de nous. (p. 540) il ne les lâcha pas durant trois mois, et ce ne fut que pour les jeter dans un combat où tous périrent ». (Miracles de Saint Ursmer : Acta Sanctorum ( $3^{\mathrm{e}}$ éd.), Aprilis II, p. 555-575, C, 5, p. 571, cité par Dominique Barthélemy dans L'an mil et la paix de Dieu, Paris, Fayard, 1999, p. 538-540). Voir également Dominique Barthélemy, « Exorciser les démons de la vengeance en Flandre autour de 1100 », dans Au cloître et dans le monde. Femmes, hommes et sociétés (IX-XV siècle). Mélanges en l'honneur de Paulette L'Hermitte-Leclercq, éd. Patrick Henriet et Anne-Marie Legras, Paris, Presses Universitaires de Paris-Sorbonne, 2000, p. 269-280.

${ }^{40}$ Claude Gauvard, "De grace especial ». Crime, État et société en France à la fin du Moyen Âge, Paris, Presses universitaires de la Sorbonne, 2010, Fac-sim. de l'édition de Paris, Publications de la Sorbonne, 1991, coll. "Histoire ancienne et médiévale », p. 872. 
son bon plaisir" ${ }^{41}$ ». Sans doute l'âme de cet homme comptait-elle parmi celles qui sèment la discorde. Comment alors trouver, au Moyen Âge, la paix intérieure?

\section{Paix intérieure et solitude}

Dès les premiers siècles du christianisme, la quête du repos de l'âme ici-bas a été envisagée à travers le retrait du monde. Les moines égyptiens comme Antoine et Pacôme ont d'abord cherché dans le désert - lieu hostile et vide d'hommes - l'éloignement des vaines tentations. D'autres ont tenté des expériences plus extrêmes. Les dendrites, ces ascètes qui vivent dans les arbres en sont un exemple. Pourtant, très vite, la solitude réelle trouve ses limites et engendre des difficultés matérielles et spirituelles qui viennent troubler le repos tant cherché. D'une part, un milieu tout à fait hostile aux hommes ne permet pas de mener cette vie sur le long terme : si les vivres et surtout l'eau viennent à trop manquer, l'expérience de la solitude tourne court devant les nécessités d'un ravitaillement minimal. D'autre part, l'isolement complet ne met pas à l'abri du démon, bien au contraire. Le Christ lui-même, après son baptême, s'est retiré dans le désert et a été tenté trois fois par le diable (Matthieu, IV, 2-11) : il a su lui résister mais les simples mortels ont rarement cette force. Le dendrite Elie le Brouteur voit ainsi sa solitude troublée par une femme assoiffée qui lui demande un peu d'eau. Après le départ de la tentatrice, le malheureux éprouvé songe à apaiser les souffrances de sa chair en un geste interdit et n'est arrêté dans son élan que par l'apparition d'un ange ${ }^{42}$. Le désert d'Elie, presque vide d'hommes, est particulièrement fréquenté par les anges et les démons, et son âme n'y connaît pas le repos. Cette solitude, loin d'apporter l'apaisement à l'âme éloignée des tentations, ne fait qu'accroître la force

\footnotetext{
${ }^{41}$ Ibid., p. 873.

42 Pierre-Louis Gatier, « Des femmes au désert? » dans Moines et religieux, éd. Jacques Berlioz, Paris, Éditions du Seuil, coll. « Points histoire », p. 171-186, p. 173.
} 
des démons intérieurs. Elle est d'ailleurs tout à fait déconseillée, voire interdite, par les pères de l'Église aux femmes dont, comme l'écrit Jérôme, la pensée « changeante et flottante [...] glisse aisément au pire ${ }^{43} »$. Face à ce constat d'échec dans la solitude la plus grande, des solutions intermédiaires sont envisagées.

Byzance connaît très tôt le phénomène des stylites et des reclus. Il s'agit dans les deux cas d'une solitude entourée et encadrée. Ces ascètes se retirent dans des lieux caractérisés par l'élévation verticale. Daniel le Stylite, au VIII ${ }^{\mathrm{e}}$ siècle, établit son habitation solitaire et en haut d'une colonne venteuse. Au milieu du XII ${ }^{\mathrm{e}}$ siècle, Néophytos dit le Reclus creuse son abri, son enkleistra, dans le flanc d'une paroi rocheuse abrupte à plus de trente-deux mètres de hauteur. L'accès, qui se fait uniquement par une échelle, est périlleux. La verticalité reste néanmoins un élément important dans la construction mentale de la distance prise par rapport au monde, tout en permettant à Néophytos et Daniel de rester proches des hommes : la caverne de Néophytos est en surplomb du monastère qu'il a fondé ; la colonne de Daniel, quant à elle, est à l'entrée de la ville. Leur ravitaillement est assuré par leurs pairs, si ces derniers ne les oublient pas, comme cela arrive parfois à Néophytos en temps de fête, lorsque les moines sont trop occupés. Néophytos et Daniel reçoivent aussi fréquemment des visiteurs qui leur demandent conseils et prières. Cependant, stylites et reclus byzantins sont pour la plupart des hommes, le plus souvent des moines, et parfois même higoumènes. En Occident, la réclusion est davantage le fait de laïcs, non seulement des hommes mais aussi des femmes. Là encore, l'isolement relatif est vu comme un bienfait. Aelred de Rievaux justifie ainsi son choix de se couper du monde auprès de sa jeune sœur pour laquelle il écrit une règle :

\footnotetext{
${ }^{43}$ Cité par Pierre-Louis Gatier, dans « Des femmes au désert ? », art. cit., p. 174.
} 
Sans doute la continence est un don de Dieu; personne n'est continent si Dieu ne lui en fait la grâce, et ce don ne doit jamais être attribué au mérite de l'homme, mais seulement au geste gratuit de Dieu. Cependant, Dieu en juge indigne ceux qui ne font aucun effort pour l'obtenir, qui s'imaginent pouvoir rester chastes au milieu des plaisirs, continents parmi les festins, qui pensent pouvoir fréquenter les jeunes filles sans être tentés, s'emplir de vapeurs excitantes dans les banquets et les beuveries et ne pas faire le mal, se lier des flammes sur la poitrine et ne pas se brûler. N'est-ce point là chose difficile, ou plutôt impossible? A toi d'en juger ${ }^{44}$.

Toutefois l'isolement induit par la réclusion, bien plus que la solitude - toute relative - n'éloigne pas tout à fait le démon qui vient tenter les recluses. La vie conventuelle, à l'abri de la clôture, présente sur ce point bien des avantages. Par clôture, il faut entendre aussi bien le mur protecteur de l'enceinte que l'ensemble des règles qui restreignent les sorties des religieux et les entrées des laïcs dans le monastère. L'architecture des lieux de clôture affiche souvent la volonté des fondateurs et des ordres de matérialiser l'éloignement du monde et d'offrir à l'âme un lieu de repos ${ }^{45}$. Rares sont les portes et les fenêtres donnant sur l'extérieur. Les sorties à l'air libre se font dans les cloîtres, à l'abri des tentations terrestres. Les règles prévoient pour la plupart l'organisation des séances du chapitre, au cours desquelles est donnée à chacun la possibilité de décharger son âme des péchés commis ; la recherche de la paix individuelle devient également

${ }^{44}$ Aelred de Rievaulx, La vie de recluse, trad. Charles Dumont, Sources chrétiennes, $\mathrm{n}^{\mathrm{o}}$ 76, Paris, 1961, p. 89, cité dans Michel Parisse, Les nonnes au Moyen Age, Le Puy, Christine Bonneton, 1983, p. 180-181.

${ }^{45}$ Gabrielle Démians d'Archimbault évoquant les cloîtres rappelle que «dans tous les cas cependant, l'on ne saurait oublier la finalité profonde de ces jardins clos, au cœur même des espaces monastiques ou séculiers : lieux de passage et de repos, mais aussi de méditation et de lecture, d'enseignement... ». Voir Gabrielle Démians d'Archimbault, «Cloître » dans Dictionnaire du Moyen Âge, dir. Claude Gauvard, Alain de Libera, Michel Zink, Paris, Presses Universitaires de France, 2002, PUF-Quadrige 2004, p. 303-304, p. 304. 
l'affaire de tous, puisque frères et sœurs ont aussi à ce moment l'occasion de dénoncer charitablement leurs pairs afin de leur permettre de recevoir la pénitence salvatrice. La règle de l'hôpital Saint-Jean-Baptiste dit Gantois à Lille, qui s'appuie sur les préceptes d'Augustin, en fournit un exemple. Les accusations portées à l'encontre d'une sœur doivent être fondées et l'accusatrice exempte de tout conflit intérieur :

Et se li une des seurs veult proclamer et accuser une aultre, elle le doit accuser par caritee, sans rancune, sans mouvement de ire et sans hayne, non point par soupechon ne par oir dire mays de choses que elle aura veu et oy et sil advient que elle accuse aultruy par oir dire elle doit dire de quy elle l'a oy ${ }^{46}$.

Le chapitre, tout en mettant en lumière les péchés de chacun(e), permet de mieux les combattre. Si la paix entre les individus est bien selon Claude Gauvard un « effort constant pour négocier, palabrer, inventer sans cesse de nouvelles formules qui contraignent les adversaires et les contiennent dans les limites fixées ${ }^{47}{ }^{\prime}$, il en va de même pour la paix intérieure.

\section{De la paix intérieure à l'hérésie}

En effet, au Moyen Âge, en Occident, la quête de la paix intérieure passe nécessairement par la contrainte de ses propres démons dans un cadre déterminé par l'Église et ne peut en aucun cas être un processus personnel de recherche, comme le montre l'évolution du mouvement des béguinages. Le mouvement des béguinages connaît en Flandre une grande expansion au $\mathrm{XIII}^{\mathrm{e}}$ siècle, soutenu à la fois par de puissants fondateurs, comme les comtesses de Flandre pour les béguinages de Lille et Gand par exemple,

\footnotetext{
${ }^{46}$ Lille, Archives départementales du Nord, AH VIII E 1, avril 1467, fol. 8 vo.

47 Claude Gauvard, "Avant-Propos », dans Le règlement des conflits au Moyen Âge, $X X X I^{e}$ Congrès de la SHMESP (Angers, juin 2000), Paris, Publications de la Sorbonne, 2001, p. 7-9, p. 8.
} 
mais aussi par les ordres mendiants. En Flandre, il s'agit essentiellement de pieuses femmes qui, sans avoir prononcé de vœux perpétuels, vivent en communauté, travaillent le jour dans le siècle et disposent chacune d'une maison dans l'enceinte protégée d'un béguinage, situé à l'extérieur proche de la ville. Ce modèle ne se trouve pas en dehors des Pays-Bas. Selon Walter Simons, le retrait hors du monde de ces béguines est avant tout une construction mentale plutôt qu'une réalité physique ${ }^{48}$. Pour l'Église, le problème est bien là : comment contrôler ce qui est une construction mentale ? Comment s'assurer de ses fondements, de ses tenants et de ses aboutissants ? Certaines sont accusées de déviances telles que le quiétisme ou une réticence à accepter les autorités ecclésiastiques. L'Église a du mal à se positionner face à ces femmes, essentiellement en quête de salut. Trois d'entre elles sont bien connues: Marie d'Oignies, Douceline de Digne et Marguerite Porrette. Si les deux premières sont béatifiées, la troisième est brûlée comme hérétique. En 1446, le pape unit les béguines et les bégards aux tertiaires des ordres mendiants. La paix intérieure terrestre ne peut donc être trouvée que dans les cadres prévus ou aménagés par l'Église, sous peine de ne pas accéder au repos de l'âme mais de toucher à l'hérésie et de mettre ainsi en danger la cohésion sociale.

\section{«Trouver » la paix ?}

L'esquisse des orientations actuelles de la recherche montre que désormais la paix est davantage envisagée comme un processus que comme un état statique ${ }^{49}$; de même, c'est à l'évolution et à la transformation des

48 Walter Simons, Cities of Ladies. Beguine Communities in the Medieval Low
Countries, 1200-1565, Philadelphie, University of Pennsylvania Press, 2001, p. 61 .
49 On peut remarquer que, de façon significative, Nicolas Offenstadt a choisi d'intituler
son ouvrage « Faire la paix au Moyen Âge » (Nicolas Offenstadt, op. cit.); en l'an 2000,
Diane Wolfthal affirmait déjà que le but de l'ouvrage dont elle avait la direction était de
se démarquer des analyses classiques de la paix comme état pour s'intéresser au
contraire à la paix comme processus dynamique (Diane Wolfthal, "Introduction », 
idées de paix que l'on s'intéresse actuellement. C'est donc bien la paix en tant que dynamique qui retient l'attention et engendre un foisonnement de publications portant sur les stratégies développées par les hommes du Moyen Âge pour atteindre la paix ${ }^{50}$.

Un tel angle d'approche soulève nécessairement une question: la paix peut-elle être «trouvée »? Plusieurs historiens ont remis en cause l'idée que cela soit effectivement possible. Par exemple, si l'on choisit de considérer la réussite d'une paix internationale en fonction du succès des accords passés par des gouvernants, on en vient facilement à penser que ces tentatives se soldent toujours par un échec. Quant aux négociations menées entre personnes de condition sociale plus humble, elles ne sont pas toujours plus efficaces : Patrick Geary souligne par exemple la reprise périodique des anciennes querelles au fur et à mesure des années ou des générations dans le royaume de France des années 1050-1200¹ . Pour Claude Gauvard, la paix est donc davantage un désir qu'une réalité : elle apparaît, selon les propres termes de l'historienne, comme «bricolée $»^{52}$.

Cette analyse pose le problème des critères que l'on choisit de retenir pour évaluer la réussite ou l'échec d'une paix. Jenny Benham s'interroge sur la durée pertinente pour juger du succès de la paix si l'on décide de s'appuyer sur sa longévité ; par ailleurs, elle se demande si la reprise des hostilités entre deux rois dans un espace donné, alors qu'un accord avait été trouvé pour un espace différent, constitue ou non une rupture de la paix entre ces deux gouvernants. Rejetant ces critères, elle propose de conclure qu'au moins pour l'Angleterre et le Danemark du XII ${ }^{\mathrm{e}}$ siècle, la réussite de

art. cit.,p. xix). La même perspective est généralement reprise dans les ouvrages publiés dans les années 2000.

${ }^{50}$ Le recueil Peace and Negotiation, par exemple, met l'accent sur la variété de ces stratégies, qu'il s'agisse de définir spatialement la paix que l'on veut mettre en œuvre, de valoriser les intérêts culturels communs ou encore d'assurer l'apaisement des tensions par les mariages. (éd. Diane Wolfthal, op. cit.).

${ }^{51}$ Patrick Geary, art. cit.

${ }^{52}$ Voir supra, note 48. 
la paix pour les contemporains tenait beaucoup plus à la façon dont cette paix était conclue qu'à sa durée. L'important était alors d'enregistrer qu'à travers des gestes et des cérémonies variables, chaque gouvernant avait rempli son devoir d'assurer la paix future et la restauration de l'ordre $\operatorname{social}^{53}$.

En ce qui concerne la paix intérieure, l'une des difficultés majeures est de savoir s'il faut envisager comme un échec ou une réussite le cas de celles et ceux qui ont trouvé seuls une forme de repos de l'âme - ou un cheminement intellectuel propre qui leur permettrait de le trouver - mais dont la démarche a été réprouvée par l'Église. La question renvoie ici à l'existence même de l'individu dans une société où le groupe prime avant tout.

Le présent bulletin souhaite proposer une perspective un peu différente. A notre sens, « trouver » ne renvoie pas uniquement - bien qu'il ne l'exclue pas totalement - à un processus de recherche mais plutôt à l'éventuelle possibilité d'atteindre un état, sans l'avoir recherché, ou à y accéder hors des sentiers habituellement prescrits pour cela, que cela soit à l'échelle du royaume, de la ville ou de l'individu. C'était en tout cas l'un des objectifs des Questeurs à l'ouverture de ce séminaire.

${ }^{53}$ Jenny Benham, op. cit., p. 207-210. 